\title{
Sciendo
}

ISSN: $1231-4005$

e-ISSN: $2354-0133$

DOI: $10.2478 /$ kones-2019-0118

\section{MODELLING OF HELICOPTER MAIN ROTOR AERODYNAMIC LOADS IN MANOEUVRES}

\author{
Grzegorz Kowaleczko \\ Polish Air Force Academy \\ Dywizjonu 303 Street 35, 08-521 Deblin, Poland \\ tel.: +48 81 5517423, fax: +48815517417 \\ e-mail: grzegorz.kowaleczko@itwl.pl \\ Andrzej Leśniczak \\ Air Force Institute of Technology \\ Ksiecia Boleslawa Street 6, 01-494 Warsaw, Poland \\ tel.: +48 261 851302, fax: +48261851313 \\ e-mail:andrzej.lesniczak@itwl.pl
}

\begin{abstract}
The article discusses the method of modelling of the helicopter main rotor aerodynamic loads during steady state flight and manoeuvres. The ability to determine these loads was created by taking into account the motion of each blade relative to the hinges and was a result of the applied method of aerodynamic loads calculating. The first part of the work discusses the basic relationships that were used to build the mathematical model of helicopter flight. The focus was also on the method of calculating of the aerodynamic forces generated by the rotor blades. The results of simulations dedicated to the "jump to hover" manoeuvre were discussed, showing the possibilities of analysing aerodynamic loads occurring in unsteady flights. The main rotor is considered separately in an "autonomous" way and treated as a source of averaged forces and moments transferred to the hub. The motion of individual blades is neglected, and their aerodynamic characteristics are radically simplified. The motion of individual blades is neglected, and their aerodynamic characteristics are radically simplified. This can lead to significant errors when attempting to model dynamic helicopter manoeuvres. The more complex model of helicopter dynamics is discussed.
\end{abstract}

Keywords: helicopter, main rotor, aerodynamic loads

\section{Introduction}

A large number of moving components of the main rotor and the tail rotor $[6,15,18,20,22$, 23] means that the mathematical model describing the helicopter's motion should take into account a sufficient number of degrees of freedom to obtain possibly full and complete description of its behaviour in different phases of flight as a result of the analysis. In addition, the complex nature of the flow around the rotor blades, variable even in steady flight conditions, makes it difficult to map all aerodynamic phenomena occurring during flight $[1-4,7-8,11,12,13-20]$.

Therefore, when modelling helicopter motion, motions of its moving parts are usually not taken into account. The rotor is considered separately in an "autonomous" way and treated as a source of averaged forces and moments transferred to the hub. The motion of individual blades is neglected, and their aerodynamic characteristics are radically simplified. This can lead to significant errors when attempting to model dynamic helicopter manoeuvres.

The more complex model of helicopter dynamics is discussed below, which eliminates the need for multiple simplifications. Thanks to the simultaneous analysis of the helicopter motion in space and the motions of the blades relative to the hinges, it was possible to determine the temporary forces acting on them and on the helicopter hub and fuselage. The applied method of calculating of these forces allows you to give up many simplifications and to take into account 
various aerodynamic phenomena, such as dynamic stall. The developed simulation model enables simultaneous analysis of helicopter dynamic manoeuvres and assessment of load variability acting on its key structural elements. Similar models of helicopter motion dynamics differing in the degree of simplification are described, among others in $[5,6,9-14,21]$.

\section{Description of the equations of motion}

\section{Coordinate systems}

The following right-handed rectangular coordinate systems shown in Fig. 1 are used in the analysis:

$O_{g} x_{g} y_{g} z_{g}-$ Earth-fixed coordinate system,

$O x_{k} y_{k} z_{k} \quad$ - fuselage-fixed coordinate system,

$P x^{\prime \prime} y^{\prime \prime} z^{\prime \prime} \quad$ - hub-fixed coordinate system,

$P_{H i} x_{i}^{\prime} y_{i}^{\prime} z_{i}^{\prime}$ - flapping hinge coordinate system,

$P_{V i} x_{i} y_{i} z_{i}$ - lag hinge coordinate system,

$O_{s o} x_{s o} y_{s o} z_{s o}$ - tail rotor hub coordinate system,

$O_{s a} x_{s i} y_{s i} z_{s i}$ - tail rotor blade coordinate system.

The conversion of vector components between these systems is performed using the transformation matrix shown in $[11,12]$.

\section{Position vectors}

To determine the equations of motion, the following position vectors are defined in Fig. 2:

h - vector determining the position of the hub centre $P$ in relation to the fuselage mass centre (the origin of the system $O x_{k} y_{k} z_{k}$ ),

$\mathbf{r}^{\prime \prime}$ - vector defining the position of the hub element relative to the centre of the hub $P$,

$\mathbf{R}^{\prime \prime}$ - vector determining the position of the hub element relative to the fuselage mass centre,

$\mathbf{l}_{H}-$ vector determining the position of the flapping hinge $\left(P_{H}\right)$ relative to the hub centre,

$\mathbf{r}^{\prime}$ - vector defining the position of the connector element relative to the flapping hinge,

$\mathbf{R}^{\prime}$ - vector determining the position of the connector element relative to the fuselage mass centre,

$\mathbf{l}_{V}-$ vector determining the position of the lag hinge $\left(P_{V}\right)$ relative to the flapping hinge,

$\mathbf{r}$ - vector defining the position of the blade element relative to the lag hinge,

$\mathbf{R}$ - vector defining the position of the blade element relative to the fuselage mass centre.

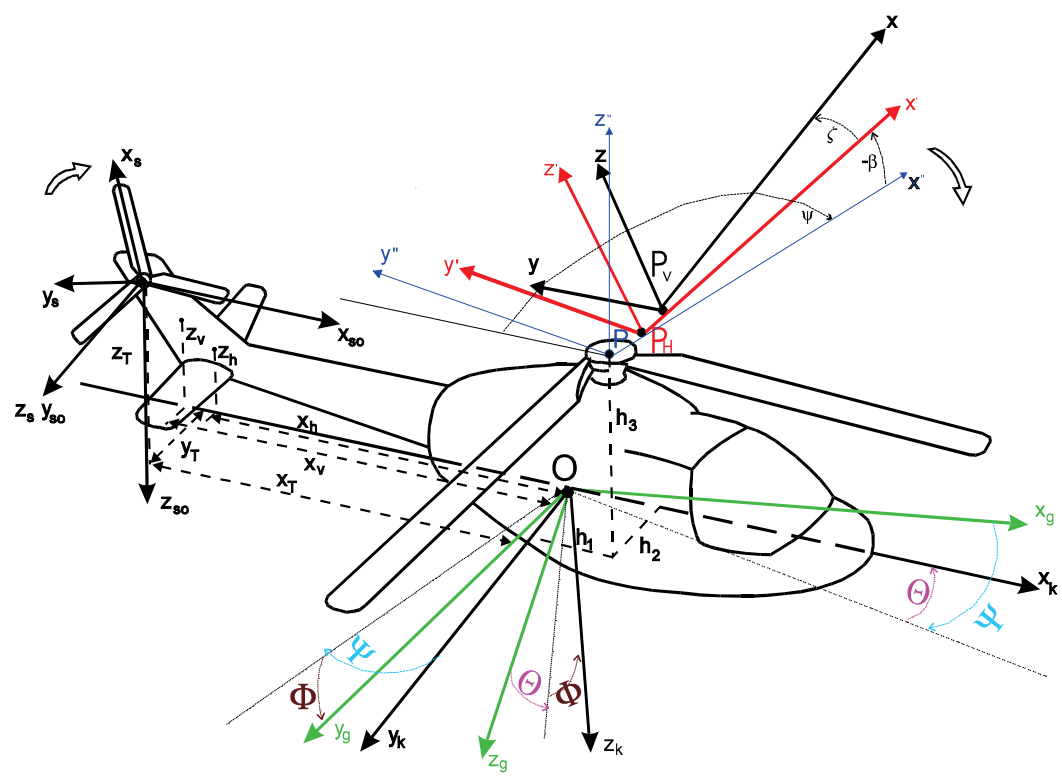

Fig. 1. Coordinate systems used to analyse helicopter motion dynamics 


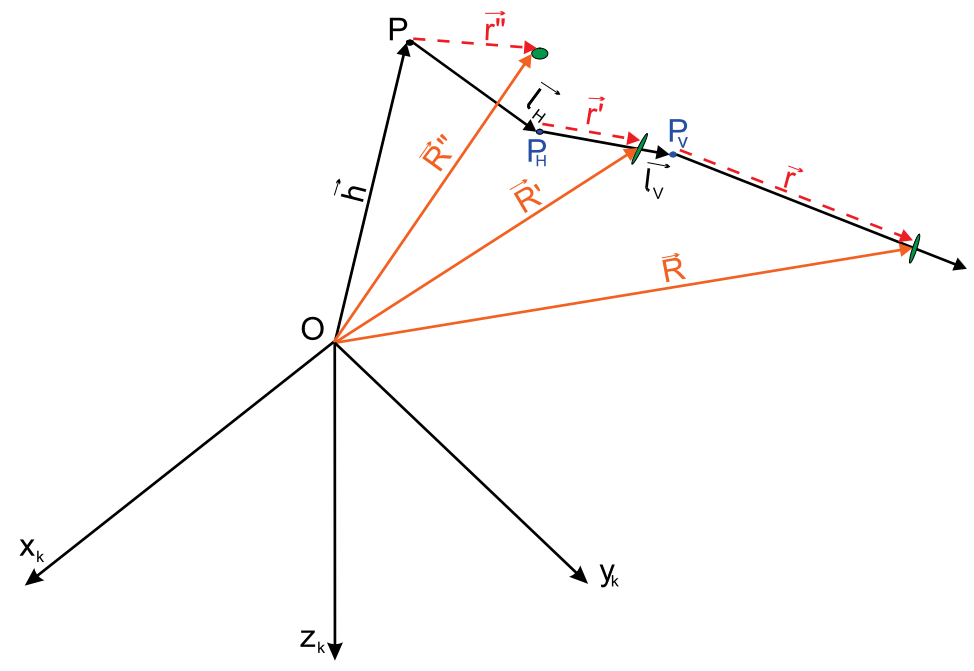

Fig. 2. Position vectors of blade, connector and hub elements (index " $i$ " is omitted)

\subsection{The force equation of motion}

Equation of linear motion of the fuselage mass centre has the form:

$$
m_{k} \frac{d \mathbf{V}_{c}}{d t}=\mathbf{F}+\mathbf{R}_{w}+\mathbf{T}_{s o}
$$

where: $m_{k}$ - fuselage mass, $\mathbf{V}_{c}-$ the absolute velocity of the fuselage mass centre, which in the $O x_{k} y_{k} z_{k}$ system has the components $\mathbf{V}_{c}=[U, V, W]^{T}, \mathbf{F}$ - the sum external forces acting on the fuselage, $\mathbf{R}_{w}$ - the force from the main rotor, $\mathbf{T}_{s o}-$ the tail rotor thrust.

Equation of the $i$-th blade element motion:

$$
\mathbf{W}_{i} d m_{i}=\mathbf{q}_{i} d r_{i}+d \mathbf{A}_{i},
$$

where: $d m_{i}$ - the mass of the element with $d r_{i}$ length, $\mathbf{W}_{i}$ - the absolute acceleration of the element, $\mathbf{q}_{i} d r_{i}=\left(\mathbf{q}_{a i}+\mathbf{q}_{g i}\right) d r_{i}$ - the vector of external forces ( $a$ - aerodynamic and $g$ - gravitational $)$ acting on the element, $d \mathbf{A}_{i}-$ the reaction of neighbouring elements.

Similar equations apply to the connector element of the $i$-th blade and the hub element:

$$
\begin{aligned}
& \mathbf{W}_{i}^{\prime} d m_{i}^{\prime}=\mathbf{q}_{i}^{\prime} d r_{i}^{\prime}+d \mathbf{A}_{i}^{\prime}, \\
& \mathbf{W}^{\prime \prime} d m^{\prime \prime}=d \mathbf{F}^{\prime \prime}+d \mathbf{A}^{\prime \prime} .
\end{aligned}
$$

Integrating equation (2) from the $P_{V i}$ lag hinge to the end of the blade (for all $k$ blades), equation (3) from the $P_{H i}$ flapping hinge to the $P_{V i}$ lag hinge (for all connectors) and equation (4) by the volume of the hub, then adding results of these integrations to equation (1), the equation of the forward helicopter motion is obtained:

$$
m_{k} \frac{d \mathbf{V}_{c}}{d t}+\sum_{i=1}^{k} \int_{P_{V i}}^{R} \mathbf{W}_{i} d m_{i}+\sum_{i=1}^{k} \int_{P_{H i}}^{P_{V_{i}}} \mathbf{W}_{i}^{\prime} d m_{i}^{\prime}+\iint_{V} \mathbf{W}^{\prime \prime} d m^{\prime \prime}=\mathbf{F}+\mathbf{T}+\mathbf{T}_{s o},
$$

where $\mathbf{T}$ is the vector of external forces acting on the rotor:

$$
\mathbf{T}=\sum_{i=1}^{k} \int_{P_{V i}}^{B R} \mathbf{q}_{i} d r_{i}+\sum_{i=1}^{k} \int_{P_{H i}}^{P_{V i}} \mathbf{q}_{i}^{\prime} d r_{i}^{\prime}+\iint_{V} d \mathbf{F}^{\prime \prime}
$$

$B$ is the tip loss factor [3]. 


\subsection{The moment equation of motion}

Equation of angular motion of the fuselage has the form:

$$
\frac{d \mathbf{K}}{d t}=\mathbf{M}+\mathbf{M}_{w}+\mathbf{M}_{s o},
$$

where: $\mathbf{M}$ - the moment of external forces acting on the fuselage, $\mathbf{M}_{w}-$ the moment of the main rotor reaction, $\mathbf{M}_{s o}$ - the moment produced by the tail rotor, $\mathbf{K}-$ the angular momentum of the fuselage.

The equation of moment's equilibrium with respect to the fuselage mass centre is obtained by adding to equation (7) sides of:

1. equation (2) multiplied vectorly by the position vector $\mathbf{R}_{i}$ and integrated from the lag hinge $P_{V i}$ to the blade end (for all blades);

2. equation (3) multiplied vectorly by the position vector $\mathbf{R}_{i}^{\prime}$ and integrated from the flapping hinge $P_{H i}$ to the lag hinge $P_{V i}$ (for all connectors);

3. equation (4) multiplied vectorly by the position vector $\mathbf{R}^{\prime \prime}$ and integrated over the hub volume.

You get:

$$
\frac{d \mathbf{K}}{d t}+\sum_{i=1}^{k} \int_{P_{V i}}^{R} \mathbf{R}_{i} \times \mathbf{W}_{i} d m_{i}+\sum_{i=1}^{k} \int_{P_{H i}}^{P_{V i}} \mathbf{R}_{i}^{\prime} \times \mathbf{W}^{\prime} d m_{i}^{\prime}+\oiiint_{V} \mathbf{R}_{i}^{\prime \prime} \times \mathbf{W}^{\prime \prime} d m^{\prime \prime}=\mathbf{M}+\mathbf{M}_{T}+\mathbf{M}_{s o},
$$

$\mathbf{M}_{T}$ is the moment of external forces acting on the rotor relative to the fuselage mass centre:

$$
\mathbf{M}_{T}=\sum_{i=1}^{k} \int_{P_{V i}}^{B R} \mathbf{R}_{i} \times \mathbf{q}_{i} d r_{i}+\sum_{i=1}^{k} \int_{P_{H i}}^{P_{V_{i}}} \mathbf{R}_{i}^{\prime} \times \mathbf{q}_{i}^{\prime} d r_{i}^{\prime}+\oiiint_{V} \mathbf{R}^{\prime \prime} \times d \mathbf{F}^{\prime \prime} .
$$

The equation determining the angular motion of the main rotor relative to the shaft axis is obtained by multiplying vectorly equations (2), (3) and (4) respectively by vectors determining the position of the elements of the blade, the connector and the hub relative to the hub centre $P$, adding the results of multiplication by sides and projecting the result of this summation onto the $P z$ " axis. The result is:

$$
\sum_{i=1}^{k} \int_{P_{V i}}^{R}\left[\left(\mathbf{l}_{H i}+\mathbf{l}_{V i}+\mathbf{r}_{i}\right) \times \mathbf{W}_{i} d m_{i}\right]_{z}{ }^{\prime}+\sum_{i=1}^{k} \int_{P_{H i}}^{P_{V i}}\left[\left(\mathbf{l}_{H i}+\mathbf{r}_{i}^{\prime}\right) \times \mathbf{W}_{i}^{\prime} d m_{i}^{\prime}\right]_{z^{\prime}}+\oiiint_{V}\left[\mathbf{r}^{\prime \prime} \times \mathbf{W}^{\prime \prime} d m^{\prime \prime}\right]_{z^{\prime \prime}}=\left[\mathbf{M}_{P}\right]_{z^{\prime \prime}}+\left[\mathbf{M}_{r k}\right]_{z}{ }^{\prime},
$$

where: $\mathbf{M}_{r k}$ means the moment of fuselage reaction equal to, under normal flight conditions, the moment from the propulsion system $\mathbf{M}_{r k}=\mathbf{M}_{p s}$, and $\mathbf{M}_{P}$ is the moment of external forces relative to the hub centre $P$. Its $P z$ "axis projection is defined as follows:

$$
\left[\mathbf{M}_{P}\right]_{z^{\prime}}=\sum_{i=1}^{k} \int_{P_{l i}}^{B R}\left[\left(\mathbf{l}_{H \hbar}+\mathbf{l}_{V i}+\mathbf{r}_{i}\right) \times \mathbf{q}_{i} d r_{i}\right]_{z^{*}}+\sum_{i=1}^{k} \int_{P_{t h}}^{P_{P_{h}}}\left[\left(\mathbf{l}_{t h}+\mathbf{r}_{i}^{\prime}\right) \times \mathbf{q}_{i}^{\prime} d r_{i}^{\prime}\right]_{z^{\prime}}+\oiiint_{V}\left[\mathbf{r}^{\prime \prime} \times d \mathbf{F}^{\prime \prime}\right]_{z^{\prime}} .
$$

In a similar way, using equations (2) and (3), the equation of motion of the $i$-th blade with respect to the flapping hinge axis $P_{H i}$ is obtained:

$$
\int_{P_{V i}}^{R}\left[\left(\mathbf{l}_{V i}+\mathbf{r}_{i}\right) \times \mathbf{W}_{i} d m_{i}\right]_{y_{i}^{\prime}}+\int_{P_{H i}}^{P_{V i}}\left[\mathbf{r}_{i}^{\prime} \times \mathbf{W}_{i}^{\prime} d m_{i}^{\prime}\right]_{y_{i}^{\prime}}=\left[\mathbf{M}_{P_{H i}}\right]_{y_{i}^{\prime}}+\left[\mathbf{M}_{\beta}\right]_{y_{i}^{\prime}} .
$$

Subscript []$_{y_{i}^{\prime}}$ means projection onto the axis $P_{H i} y_{i}^{\prime}$ of this hinge.

$\left[\mathbf{M}_{P_{H i}}\right]_{y_{i}^{\prime}}$ is the projection of moments of external forces relative to the $i$-th flapping hinge $P_{H i} y_{i}^{\prime}$ onto the axis of this hinge: 


$$
\left[\mathbf{M}_{P_{H i}}\right]_{y_{i}^{\prime}}=\int_{P_{V i}}^{B R}\left[\left(\mathbf{l}_{V i}+\mathbf{r}_{i}\right) \times \mathbf{q}_{i} d r_{i}\right]_{y_{i}^{\prime}}+\int_{P_{H i}}^{P_{V i}}\left[\mathbf{r}_{i}^{\prime} \times \mathbf{q}_{i}^{\prime} d r_{i}^{\prime}\right]_{y_{i}^{\prime}}
$$

and $\left[\mathbf{M}_{\beta}\right]_{y_{i}^{\prime}}$ takes into account damping and stiffness in the flapping hinge (Fig. 1):

$$
\left[\mathbf{M}_{\beta}\right]_{y_{i}^{\prime}}=-c_{\beta} \dot{\beta}_{i}-k_{\beta} \beta_{i} .
$$

Based on equation (2), the equation of the angular motion of the $i$-th blade in relation to the lag hinge axis $P_{V i}$ was determined:

$$
\int_{P_{V i}}^{R}\left[\mathbf{r}_{i} \times \mathbf{W}_{i} d m_{i}\right]_{z_{i}}=\left[\mathbf{M}_{P_{V i}}\right]_{z_{i}}+\left[\mathbf{M}_{\zeta}\right]_{z_{i}} .
$$

The subscript []$_{z_{i}}$ symbolizes the projection onto the $P_{V i} z_{i}$ axis of this hinge.

$\left[\mathbf{M}_{P_{V i}}\right]_{z_{i}}$ is the projection of the moments of external forces relative to the $i$-th lag hinge:

$$
\left[\mathbf{M}_{P_{V i}}\right]_{z_{i}}=\int_{P_{V i}}^{B R}\left[\mathbf{r}_{i} \times \mathbf{q}_{i} d r_{i}\right]_{z_{i}}
$$

and $\left[\mathbf{M}_{\zeta}\right]_{z_{i}}$ takes into account damping and stiffness in the lag hinge (Fig. 1):

$$
\left[\mathbf{M}_{\zeta}\right]_{z_{i}}=-c_{\zeta} \dot{\zeta}_{i}-k_{\zeta} \zeta_{i}
$$

Equations (5), (8), (10), (12) and (15) describe the spatial motion of a single-rotor helicopter, taking into account the rotational motion of the rotor and the motion of individual blades about hinges $P_{H i}$ and $P_{H i}$.

\subsection{The final form of the equations of motion}

After supplementing the above equations with kinematic relations that allow calculating the rate of change of angles $\Psi, \Theta$ and $\Phi$ determining the angular position of the helicopter relative to the inertial system and the linear velocity in the inertial system:

$$
\begin{aligned}
& \dot{\Phi}=P+(Q \sin \Phi+R \cos \Phi) \tan \Theta, \\
& \dot{\Theta}=Q \cos \Phi-R \sin \Phi, \\
& \dot{\Psi}=(R \cos \Phi+Q \sin \Phi) / \cos \Theta, \\
& {\left[\begin{array}{c}
U_{g} \\
V_{g} \\
W_{g}
\end{array}\right]=\left[\begin{array}{l}
\dot{x}_{g} \\
\dot{y}_{g} \\
\dot{z}_{g}
\end{array}\right]=\hat{\boldsymbol{\alpha}}^{-1}\left[\begin{array}{c}
U \\
V \\
W
\end{array}\right]}
\end{aligned}
$$

and considering that:

$$
\frac{d \beta_{i}}{d t}=\dot{\beta}_{i}, \frac{d \zeta_{i}}{d t}=\dot{\zeta}_{i}, \frac{d \psi}{d t}=\omega,
$$

the $14+4$ nonlinear ordinary differential equations were obtained, which can be written as:

$$
\mathbf{A}(t, \mathbf{x}) \dot{\mathbf{x}}+\mathbf{B}(t, \mathbf{x})=\mathbf{f}(t, \mathbf{x}, \mathbf{s}),
$$

where $\mathbf{x}$ is a flight parameter vector: 


$$
\mathbf{x}=\left[U, V, W, P, Q, R, \omega, \dot{\beta}_{i}, \dot{\zeta}_{i}, \beta_{i}, \zeta_{i}, \psi, \Phi, \Theta, \Psi, x_{g}, y_{g}, z_{g}\right]^{T},
$$

$\mathbf{s}$ is the control vector, which consists of the angles determined with the swash plate and the collective pitch angle of the tail rotor:

$$
\mathbf{s}=\left[\theta_{0}, \theta_{s}, \theta_{c}, \varphi_{s o}\right]^{T} .
$$

The vector $\mathbf{f}$ represents forces and moments acting on the fuselage and the helicopter's moving parts taken into account in the model, as well as the right sides of (18) and (19).

The effective solution of these equations requires the calculation of the absolute velocities and absolute accelerations of individual structural elements. For example, for a blade element we have:

- the absolute velocity:

$$
\mathbf{V}_{i}=\mathbf{V}_{c}+\mathbf{\Omega} \times \mathbf{R}_{i}+\boldsymbol{\omega} \times\left(\mathbf{l}_{H i}+\mathbf{l}_{V i}+\mathbf{r}_{i}\right)+\dot{\boldsymbol{\beta}}_{i} \times\left(\mathbf{l}_{V i}+\mathbf{r}_{i}\right)+\dot{\zeta}_{i} \times \mathbf{r}_{i},
$$

where $\mathbf{V}_{c}$ is the helicopter mass centre velocity, $\boldsymbol{\Omega}$ - the fuselage angular velocity.

The last three components in (21) give the relative velocity:

$$
\mathbf{V}_{r i}=\boldsymbol{\omega} \times\left(\mathbf{l}_{H i}+\mathbf{l}_{V i}+\mathbf{r}_{i}\right)+\dot{\boldsymbol{\beta}}_{i} \times\left(\mathbf{l}_{V i}+\mathbf{r}_{i}\right)+\dot{\zeta}_{i} \times \mathbf{r}_{i},
$$

$\boldsymbol{\omega}$ - the angular velocity of the main rotor, $\dot{\boldsymbol{\beta}}_{i}-$ the flapping motion angular velocity, $\dot{\zeta}_{i}-$ the lagging motion angular velocity,

- the absolute acceleration:

$$
\mathbf{W}_{i}=\frac{d \mathbf{V}_{i}}{d t}=\mathbf{W}_{c}+\frac{d \boldsymbol{\Omega}}{d t} \times \mathbf{R}_{i}+\boldsymbol{\Omega} \times\left(\boldsymbol{\Omega} \times \mathbf{R}_{i}\right)+\frac{d^{\prime} \mathbf{V}_{r i}}{d t}+\left(\boldsymbol{\omega}+\dot{\boldsymbol{\beta}}_{i}+\dot{\zeta}_{i}\right) \times \mathbf{V}_{r i}+2 \boldsymbol{\Omega} \times \mathbf{V}_{r i}
$$

\section{Forces and moments acting on the helicopter}

The helicopter is subjected to aerodynamic forces and moments (subscript $a$ ) and gravitational (subscript $g$ ) and produced by the tail rotor (subscript so). Following these indications, the following can be written:

- the vector of external forces acting on the fuselage:

$$
\mathbf{F}=\mathbf{F}_{a}+\mathbf{F}_{g}+\mathbf{T}_{s o},
$$

- the vector of external forces acting on the main rotor:

$$
\mathbf{T}=\mathbf{T}_{a}+\mathbf{T}_{g},
$$

- the moment of external forces acting on the rotor relative to the fuselage mass centre:

$$
\mathbf{M}_{T}=\mathbf{M}_{T a}+\mathbf{M}_{T g},
$$

- the moment of external forces acting on the fuselage:

$$
\mathbf{M}=\mathbf{M}_{a}+\mathbf{M}_{g}+\mathbf{M}_{s o},
$$

- the moment of external forces relative to the rotor shaft axis $P Z^{\prime \prime}$ :

$$
\mathbf{M}_{P}=\mathbf{M}_{P a}+\mathbf{M}_{P g},
$$

- the moment of external forces relative to the the axis $P_{H i} y_{i}^{\prime \prime}$ of the $i$-th flapping hinge:

$$
\mathbf{M}_{P_{H i}}=\mathbf{M}_{P_{H i} a}+\mathbf{M}_{P_{H i} g},
$$


- the moment of external forces relative to the axis $P_{V i} z_{i}$ of the $i$-th lag hinge:

$$
\mathbf{M}_{P_{V i}}=\mathbf{M}_{P_{V i} a}+\mathbf{M}_{P_{V i} g} \text {. }
$$

\subsection{Aerodynamic forces and moments}

Calculation of aerodynamic forces and moments acting on the fuselage is done based on knowledge of aerodynamic coefficients, using classic expressions:

$$
\begin{gathered}
P_{x a k}=C_{x a k} \frac{\rho\left|\mathbf{V}_{c}\right|^{2}}{2} S_{w N}, \quad P_{y a k}=C_{y a k} \frac{\rho\left|\mathbf{V}_{c}\right|^{2}}{2} S_{W_{N}}, \quad P_{z a k}=C_{z a k} \frac{\rho\left|\mathbf{V}_{c}\right|^{2}}{2} S_{W_{N}}, \\
L_{a k}=C_{l k} \frac{\rho\left|\mathbf{V}_{c}\right|^{2}}{2} S_{W_{N}} R, \quad M_{a k}=C_{m k} \frac{\rho\left|\mathbf{V}_{c}\right|^{2}}{2} S_{W_{N}} R, \quad N_{a k}=C_{n k} \frac{\rho\left|\mathbf{V}_{c}\right|^{2}}{2} S_{W N} R,
\end{gathered}
$$

$S_{W N}, R$ - rotor area and radius, respectively.

All aerodynamic forces and moments produced by blades were calculated by integrating the appropriate expressions along their spans. For example, the aerodynamic moment relative to the flapping hinge is:

$$
\left[\mathbf{M}_{P_{H i} a}\right]_{y_{i}^{\prime}}=-\int_{P_{V i}}^{B R} q_{z a i}\left(l_{V}+r_{i} \cos \zeta_{i}\right) d r_{i}-\int_{P_{H i}}^{P_{V i}} q_{z a i}^{\prime} r_{i}^{\prime} d r_{i}^{\prime},
$$

and the aerodynamic moment relative to the lag hinge:

$$
\left[\mathbf{M}_{P_{V i} a}\right]_{z_{i}}=\int_{P_{V i}}^{B R} r_{i} q_{x a i} d r
$$

$B$ is the tip loss factor of the main rotor. $q_{x a i}$ i $q_{z a i}$ are the aerodynamic loads of the blade element. According to Fig. 3, they are equal to:

$$
q_{z a i}=d P_{z a} \cos \alpha^{*}+d P_{x a} \sin \alpha^{*}, \quad q_{y a i}=d P_{x a} \cos \alpha^{*}-d P_{z a} \sin \alpha^{*},
$$

$d P_{z a}$ i $d P_{x a}$ are components of aerodynamic forces calculated on the basis of classic expressions:

$$
d P_{z a}=C_{z a} \frac{\rho|\mathbf{V}|^{2}}{2} b(r), \quad d P_{x a}=C_{x a} \frac{\rho|\mathbf{V}|^{2}}{2} b(r),
$$

where $b(r)$ is the aerodynamic chord of the blade.

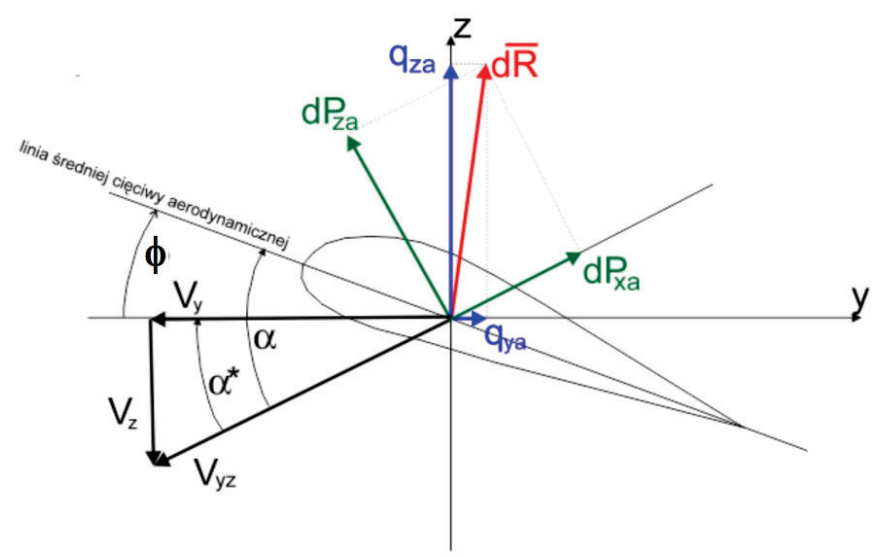

Fig. 3. Aerodynamic loads of the rotor blade element 
The calculation of these forces requires knowledge of the aerodynamic characteristics of the airfoil in the full range of the angle of attack $0-360^{\circ}$ and components of the local velocity vector. This vector is the sum of the velocity resulting from the blade motion (21) and the induced velocity vector:

$$
\mathbf{V}=\mathbf{V}_{i}+\mathbf{v}_{\text {ind }} .
$$

The induced velocity can be modeled in different ways. In simulations, this velocity was calculated based on the Biot-Savart law, taking into account the spatial position of vortices (Fig. 4), which were generated by blades at earlier moments of time:

$$
d \mathbf{v}_{\text {ind }}=\frac{\Gamma}{4 \pi} \frac{d \mathbf{s} \times \mathbf{l}}{\mid \mathbf{l}^{3}} .
$$

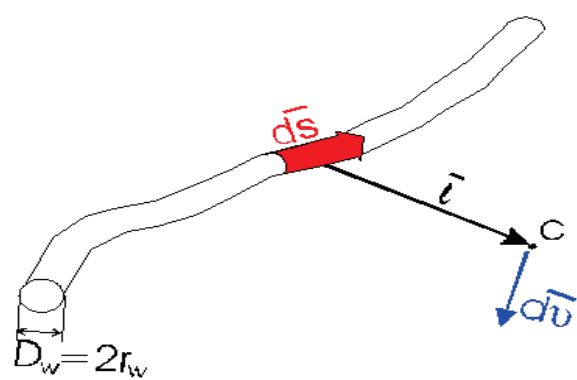

Fig. 4. Determination of the induced speed from the vortex element

The rotor blades are controlled by changing the blade angle $\varphi$ shown in Fig. 3 using the swash plate mechanism. It can be described as follows:

$$
\varphi=\theta_{0}-\theta_{s} \sin \psi_{i}-\theta_{c} \cos \psi_{i}+\kappa \beta_{i}+\varphi_{r}\left(r_{i}\right) .
$$

This formula includes swash plate control angles $\theta_{0}, \theta_{s}, \theta_{c}$, flapping compensation $\kappa \beta_{i}$ and blade geometric torsion $\varphi_{r}\left(r_{i}\right)$.

\subsection{Mass forces and moments}

The mass forces and moments occurring in formulas (24)-(30) are equal to:

- the helicopter weight:

$$
\mathbf{F}_{g}=m \mathbf{g},
$$

- the moment of fuselage mass forces relative to its mass centre:

$$
\mathbf{M}_{g}=0 \text {, }
$$

- the moment of mass forces acting on the rotor relative to the fuselage mass centre:

$$
\mathbf{M}_{T g}=\sum_{i=1}^{k} \int_{P_{V i}}^{B R} \mathbf{R}_{i} \times \mathbf{q}_{g i} d r_{i}+\sum_{i=1}^{k} \int_{P_{H i}}^{P_{V_{i}}} \mathbf{R}_{i}^{\prime} \times \mathbf{q}_{g i}^{\prime} d r_{i}^{\prime}+\int \oiiint_{V} \mathbf{R}^{\prime \prime} \times d \mathbf{F}_{g}^{\prime \prime} .
$$

The projection of mass forces, acting on the rotor, onto shaft axis $P z^{\prime \prime}$ is calculated similarly to (42) replacing the position vectors $\mathbf{R}$ with vectors specifying the position of the considered elements, relative to the hub centre - e.g. for the blade element $\left(\boldsymbol{l}_{\mathrm{H}}+\boldsymbol{l}_{\mathrm{V}}+\mathbf{r}\right)$. The moment of mass forces relative to the axis of flapping hinge must take into account the position vectors of the connector and the blade elements relative to this hinge - e.g. for the blade element $\left(\boldsymbol{l}_{\mathrm{V}}+\mathbf{r}\right)$, while the moment relative to the flapping hinge - the vector $\mathbf{r}$. 


\section{Exemplary results of calculations}

The developed model of the helicopter motion was originally used to model various flight states. The focus was on simulation of steady and manoeuvring flights by analysing helicopter motion in space and blade oscillations. The simulation software also gives the opportunity to monitor a number of parameters that are subject to dynamic changes both during steady state flight and during manoeuvres. For example, it is possible to assess how change: - the angles of attack in the plane of the rotor, the forces generated by blades and transferred to the hub.

Figures 5-8 show the basic flight parameters during the jump to the hover manoeuvre. To check the correctness of helicopter stabilization by an autopilot, it was assumed that for the first 10 seconds of flight a horizontal flight at a speed of $250 \mathrm{~km} / \mathrm{h}$ is performed. Then begins the manoeuvre consisting in a rapid increase in flight altitude (Fig. 5) with simultaneous deceleration of speed (Fig. 6). During the manoeuvre, the fuselage nose is strongly upright (Fig. 7). The maximum value of the pitch angle $\Theta$ is about $60^{\circ}$. Due to the mutual coupling of the longitudinal and lateral motions of the helicopter, rolling and yawing motions are also observed (angles $\Phi$ and $\Psi$ ). Blade motions relative to hinges are also modelled. They are shown in Fig. 8. It can be seen that by the time the manoeuvre began, the oscillations are stable. However, during manoeuvre their amplitudes change quickly. These curves allow you to assess whether there is a risk of blades hitting the swing limiters.

As it was mentioned earlier, it is also possible to obtain "maps" of distributions, e.g. angles of attack. They are shown in Fig. 9-12 for the following main rotor revolutions numbers: 43, 48, 52, 65. The corresponding fuselage pitch angles are shown in Fig. 7. Revolution number 43 refers to the conditions of the flight determined before the manoeuvre, and further successive phases of the manoeuvre. Fig. 9 shows the reverse flow zone. In other regions of the main rotor, the angles of attack have moderate values. During the manoeuvre, areas of large angles of attack appear (Fig. 10 and 11), where the critical value is exceeded. After the manoeuvre (Fig. 12), the values of the angles of attack in the entire plane of the rotor disc return to the operating range. Due to the deceleration of flight speed, the reverse flow area also disappears. Fig. 13 illustrates the distribution of attack angles along the blade on azimuths $0^{\circ}, 90^{\circ}, 180^{\circ}, 270^{\circ}$ for revolution 48 . For the returning blade $\left(270^{\circ}\right)$, no large negative values of this angle (reverse flow area) are shown. Visible strong local changes of the curves are the result of blade cutting through the tip vortices that were previously generated by all blades. Fig. 14 shows the change in the attack angles of selected blade radii during the manoeuvre. It confirms that in certain phases of the flight the critical value was exceeded (approx. $18^{\circ}$ ).

Figures 15-18 show "maps" of distribution of lift generated by blades for the same rotor revolutions. For the revolution 43, the effect of the reverse flow around the returning blade is visible. Fig. 16 and 17 confirm that cutting by the blades of the tip vortices leads to strong local changes of the angle of attack and the generated lift, while exceeding the critical angle of attack causes the decrease of the lift. Deceleration of the helicopter leads to the disappearance of the reverse flow zone (Fig. 18). Vortex cutting leads to strong disturbances in lift distribution along the blade, which results from the curves shown in Fig. 19. This applies in particular to the lapping blade $\left(90^{\circ}\right)$. Such dynamic changes can lead to strong blade structure stresses. Fig. 20 shows how the lift force changes for the three selected blade radii during the manoeuvre. Its analysis allows determining the maximum lift values. This can be useful in strength analysis.

\section{Summary}

The helicopter motion dynamics model, discussed in the article, was originally developed for the needs of flight simulation, provides great opportunities for analysing aerodynamic and mass loads occurring during shunting flights. Due to the fact that it is possible to model real flight conditions and real manoeuvres, and due to the omission of fundamental simplifications in the field of aerodynamics, the obtained results are more reliable than, e.g. obtained during the analysis of the work of the isolated rotor. 


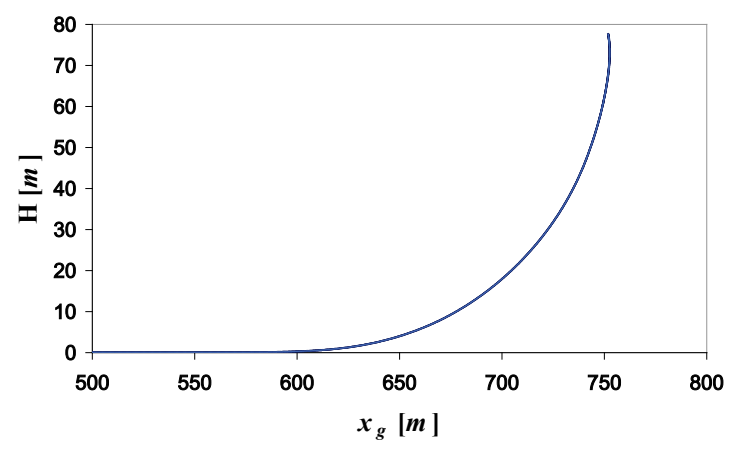

Fig. 5. Flight trajectory

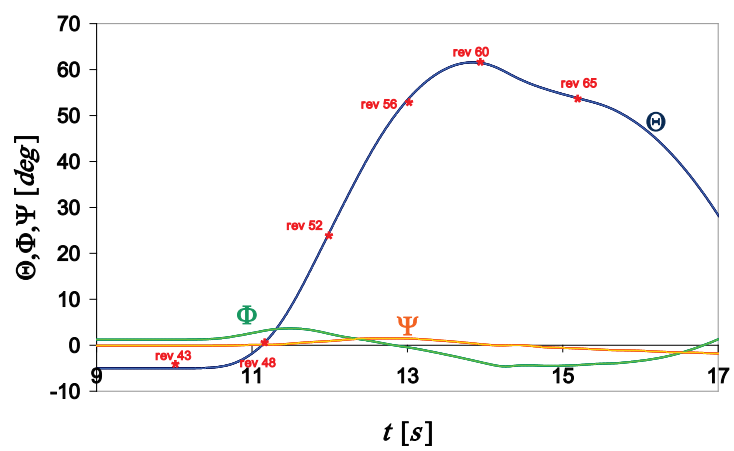

Fig. 7. Fuselage position angles

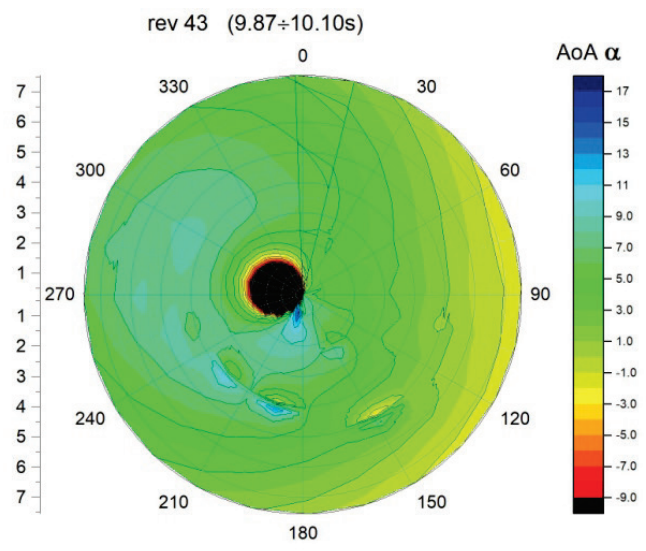

Fig. 9. Angles of Attack - rev. 43

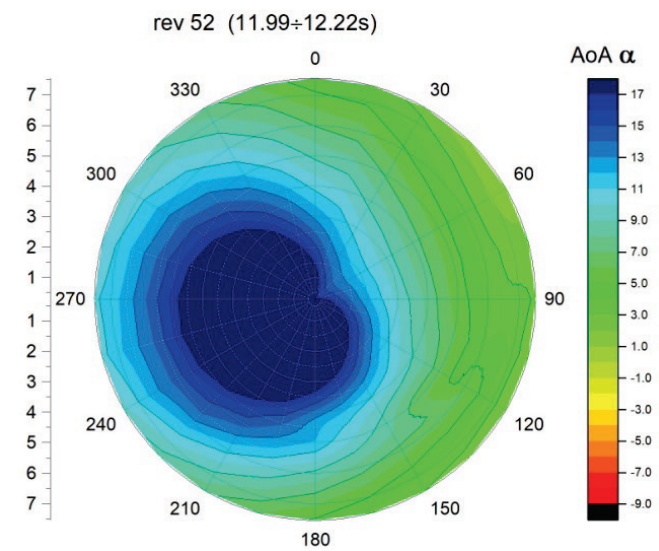

Fig. 11. Angles of Attack-rev. 52

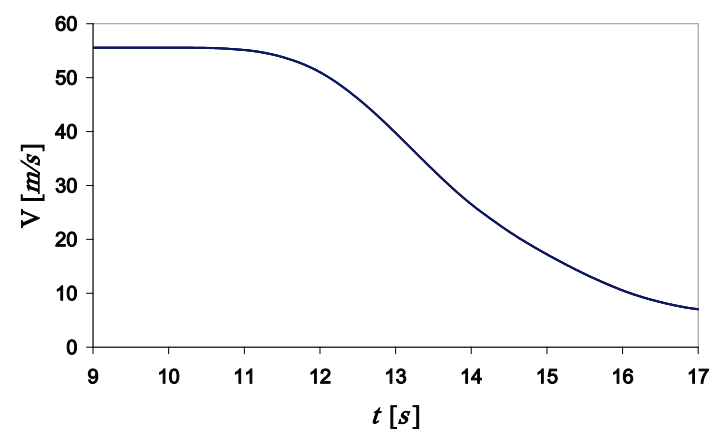

Fig. 6. Helicopter flight velocity

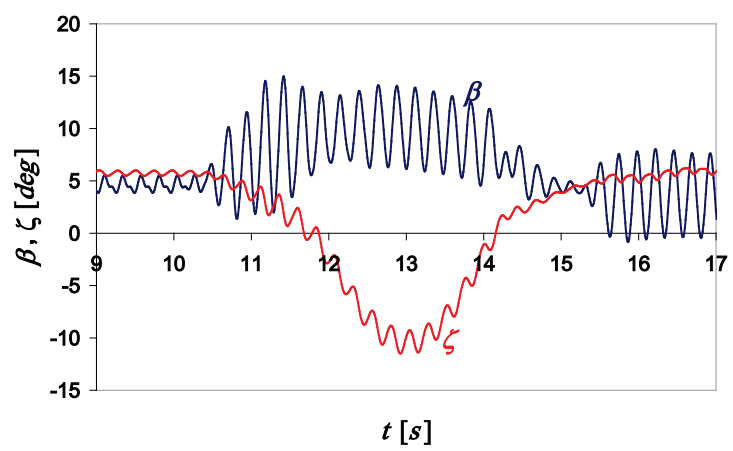

Fig. 8. Flapping and lagging angles

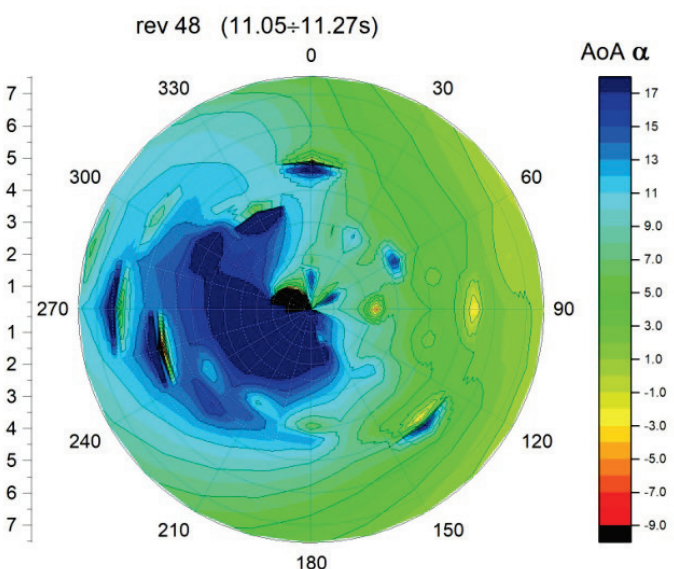

Fig. 10. Angles of Attack - rev. 48

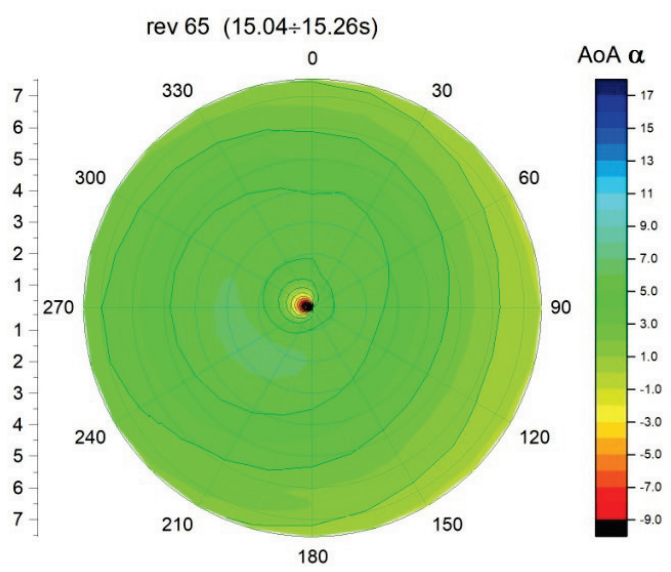

Fig. 12. Angles of Attack-rev. 65 


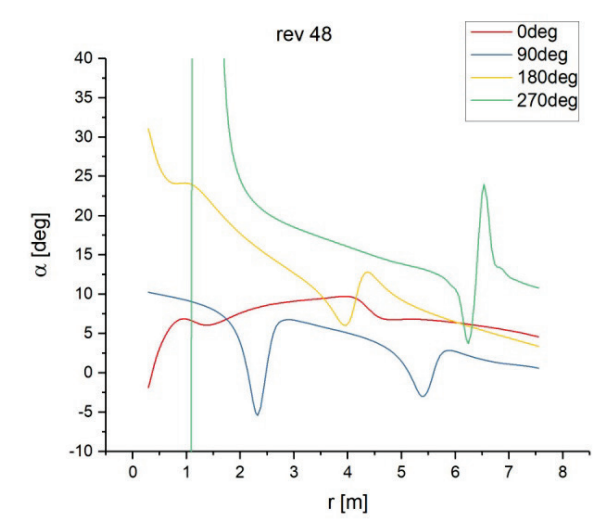

Fig. 13. A-o-A distribution along blades

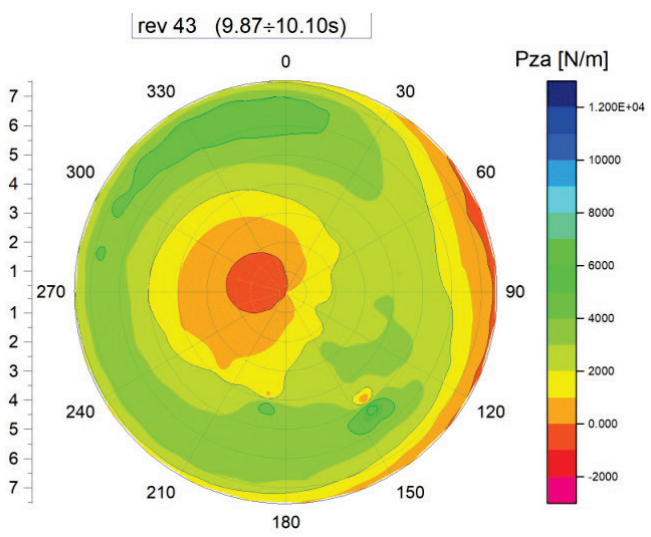

Fig. 15. Lift - rev. 43

rev $52(11.99 \div 12.22 \mathrm{~s})$

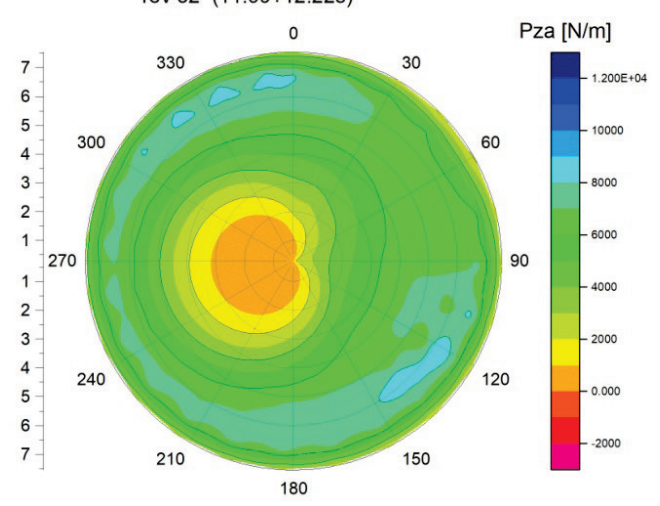

Fig. 17. Lift-rev. 52

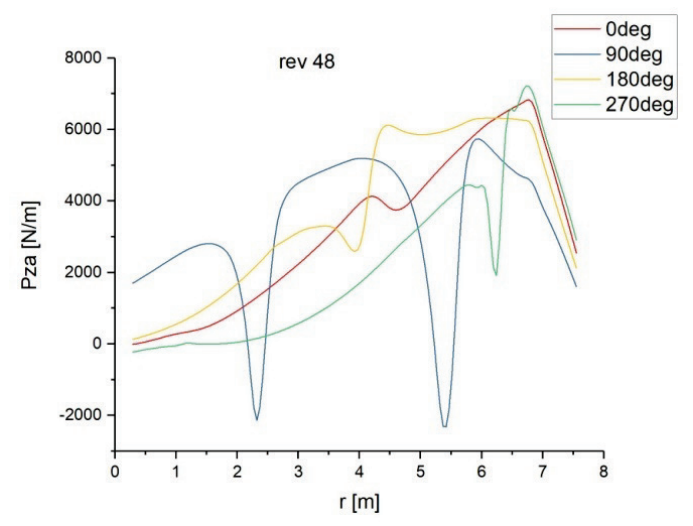

Fig. 19. Lift distribution along blades

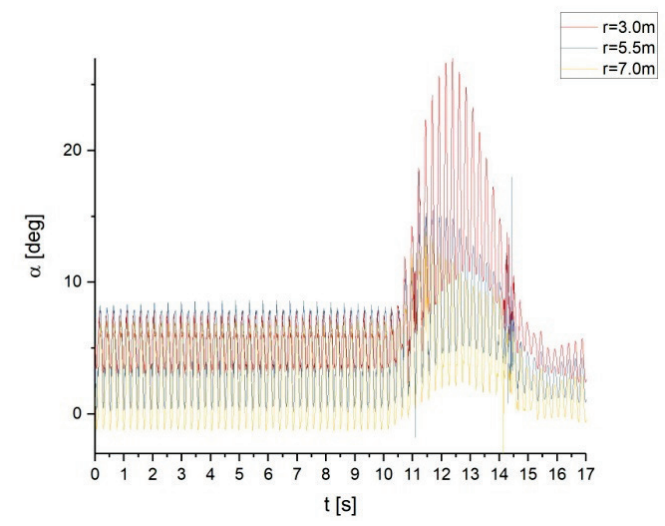

Fig. 14. Changes of A-o-A for selected radii

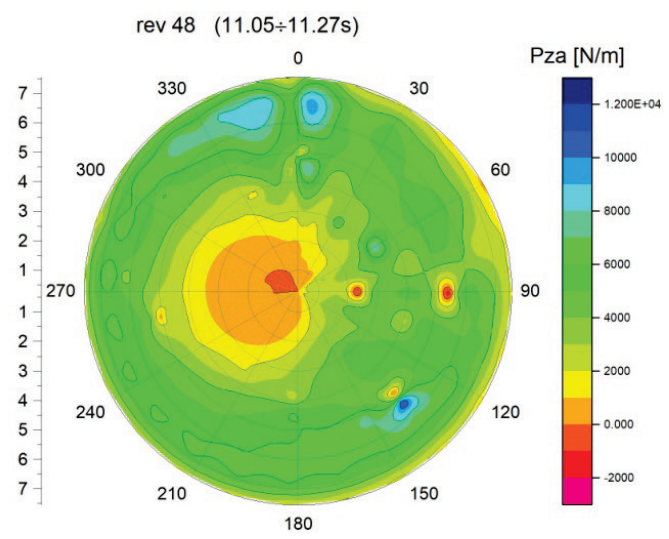

Fig. 16. Lift-rev. 48

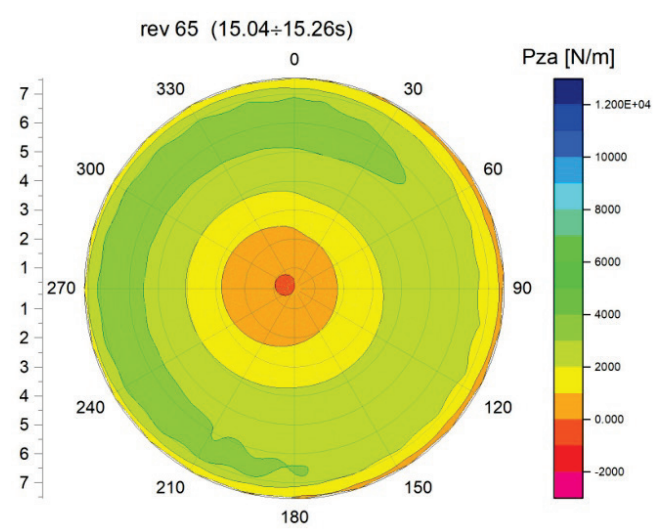

Fig. 18. Lift-rev. 65

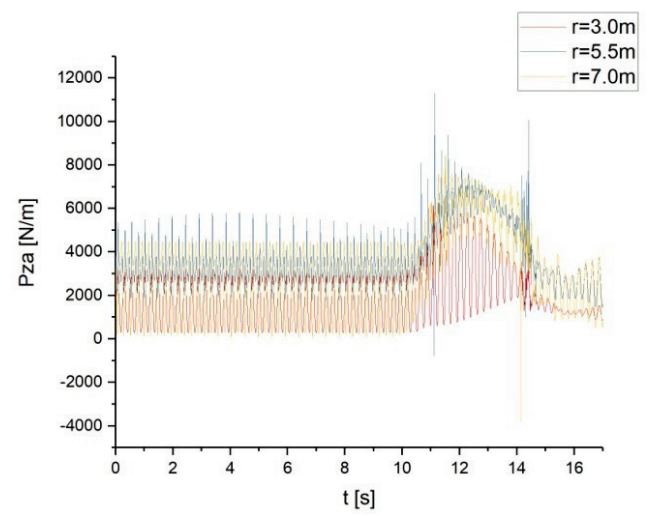

Fig. 20. Changes of lift for selected radii 


\section{References}

[1] Bazow, D. I., Helicopter Aerodynamics, "Mashinostroyenie" Publishing House, Moscow 1969.

[2] Bieriestow, L. M., Modeling of Helicopter Dynamics in Flight, "Mahinostroyenie" Publishing House, Moscow 1978.

[3] Bramwell, A. R. S., Helicopter Dynamics, Edward Arnold Publishers Ltd., London 1986.

[4] Ćwik, D., Kowalski, M., Steżycki, P., Impact of work of turbine adaptive engines for the natural environment, Journal of KONES, DOI: 10.5604/01.3001.0012.7848, Vol. 25, No. 4, pp. 509-516, Warsaw 2018.

[5] Garrard, W. L., Liebst, B., Design of a Murtivariable Helicopter Flight Control Qualities Enhancement, Journal of the American Helicopter Society, Vol. 4, No. 4, 1990.

[6] Gessow, A., Aerodynamics of the Helicopter, Frederick Ungar Publishing Co., New York 1985.

[7] Jankowski, A., Kowalski, M., Start-up Processes' Efficiency of Turbine Jet Engines, Journal of KONBiN, DOI 10.1515/jok-2016-0041, No. 40, pp. 63-72, 2016.

[8] Jankowski, K., Physical and Mathematical Modeling of Helicopter Dynamic Spatial Motion, Ph.D. Thesis, Warsaw University of Technology, Warsaw 1982.

[9] Jesaułow, S. J. et all., Helicopter as a Control Object, "Mashinostroyenie" Publishing House, Moscow, 1977.

[10] Kim, F. D., Celi, R., Tischler, M., High-Order State Space Simulation Models of Helicopter Flight Mechanics, Journal of the American Helicopter Society, Vol. 38/4, 1993.

[11] Kowaleczko, G., Analysis of the Spatial Dynamics of Helicopter Motion Taking Into Account the Influence of Autopilot, Ph.D. Thesis, Military University of Technology, Warsaw 1992.

[12] Kowaleczko, G., Inverse Problem in Aircraft Flight Dynamics, Military University of Technology, Warsaw 2003.

[13] Kowaleczko, G., Modeling of Dynamics of Flying Objects, Publishing House of the Air Force Institute of Technology, Warsaw 2018.

[14] Kowaleczko, G., Nonlinear Dynamics of Helicopter Spatial Motion, Military University of Technology, Warsaw 1998.

[15] Kowalski, M., Application of the phase reproduction method to the analysis of an avionic event on board of the W-3 "Sokót" helicopter, Journal of KONES, Vol. 18, No. 1, pp. 301310, Warsaw 2011.

[16] Kowalski, M., Noise emission level versus the structure of the aircraft turbine engine, Journal of KONES, DOI: 10.5604/12314005.1138603, Vol. 19, No. 4, pp. 325-332, Warsaw 2012.

[17] Kowalski, M., Waślicki, P., Żak, J., Selected aspects of the maintenance system optimisation of the Polish Armed Forces helicopters, Journal of KONES, DOI: 10.5604/01.3001.0012.4320, Vol. 25, No. 3, pp. 99-109, Warsaw 2018.

[18] Mil, M. L., et al., Helicopters - Calculation and Design, "Mahinostroyenie" Publishing House, Moscow 1966.

[19] Padfield, G. D., Helicopter Flight Dynamics, Blackwell Publishing Ltd., 2007.

[20] Payne, P. R., Helicopter Dynamics and Aerodynamics, Sir Isaac Pitman and Sons Ltd., London 1959.

[21] Rutherford, S., Thomson, D., Helicopter Inverse Simulation Incorporating an Individual Blade Rotor Model, $20^{\text {th }}$ Congress of the International Council of the Aeronautical Sciences, ICAS-96-1.2.1.

[22] Szabelski, K., et all., Introduction to Helicopter Construction, Transport and Communication Publishers, Warsaw 1995.

[23] Szumański, K., Pilot-Helicopter Transgression, Habilitation Thesis, Warsaw University of Technology, Warsaw 1986. 